\title{
The Research of AC-Pulse Iontophoresis Apparatus
}

\author{
Man Su ${ }^{\mathrm{a}}$, Caixiang Yue and Lili Wang \\ Hebei Chemical and Pharmaceutical College, Shijiazhuang 050000, China \\ ahalf_summer81@163.com
}

Keywords: Iontophoresis, AC-pulse, Electroporation.

\begin{abstract}
Iontophoresis apparatus is a non-invasive device used to enhance the delivery of permeation across human epidermal membrane or extract endogenous substances from the body by applying a current across the membrane. This paper analyzes the transport theory of iontophoresis, and completes the whole frame design. It involved three points:1) an initial applied electroporation used to decrease the human skin electrical conductance to a target level.2)Utilize the one-chip computer to control the gain auto control circuit, make it produce a variable AC pluse, which can adjust by itself with the applying current to maintain the human skin electrical conductance at the target level during the transport study, and 3)a low voltage D.C. offset applied simultaneously with the AC to assist permeating transport. It is proved in the experiment that the skin resistance could keep constant due to the adjusting of the A.C. volt acted on the skin. Because the working current and the flux of the medicine can be constant if the skin resistance is constant, so this apparatus provide a new method for precise flux control therapy.
\end{abstract}

\section{Introduction}

The electroporation and AC-pulse are used to the ac-pulse iontophoresis apparatus as design basis, and the single-chip microcomputer control system is used for a constant flow of drugs.

\section{The theory basis of AC-pulse iontophoresis apparatus}

Human skin model. There are two kinds of drug percutaneous absorption way, one kind is through the stratum corneum and active skin leather; another way is accessory organs of the skin.

When using the method of iontophoresis assist drug percutaneous absorption, there is the third way: a new induction channel. This channel is due to the applied voltage to the skin produced electroporation, thus on the surface of the skin produces transient recoverable hydrophilic channel.

Define the factors of constant flow. In the process of iontophoresis, there are a lot of factors influencing the molecular motion to consider. In order to calculate drugs flow in advance, you need to know these factors, which affect the drug flow and have the right understanding of the interactions between them.

During the period of iontophoresis, the instantaneous flow of ions can be expressed by $\mathrm{J}_{\Psi}{ }^{[1]}$ :

$$
J_{\Psi}=\varepsilon\left\{-H^{\prime} D\left[\frac{d c}{d x}+\frac{c z F}{R_{g a s} T} \frac{d_{\Psi}}{d x}\right] \pm W^{\prime} v c\right\} .
$$

$\Psi:$ Percutaneous electric potential on the membranes of the skin epidermis

F: Faraday constant Rgas: Atmospheric constant

$\mathrm{T}$ : Temperature $\mathrm{V}$ : the average speed of electroosmosis flow

$\varepsilon$ : comprehensive factors of the skin epidermis membrane permeability and bending degrees

$\mathrm{H}$ ': obstacle factors of synchronous brown proliferation and migration caused by electric field

W ': obstacle factor of iontophoresis seepage discharge

$\mathrm{C}, \mathrm{x}, \mathrm{z}, \mathrm{D}$ : concentration, the location of the membrane, charge number, the diffusion coefficient of permeability

$\mathrm{H}$ ' can be described by the following formula ${ }^{[2]}$ :

$H^{\prime}(\lambda)=(1-\lambda 2)(1-2.104 \lambda+2.09 \lambda 3-0.948 \lambda 5)$ 
$\lambda$ : the ratio of radius of the solute and hole radius

According to type 1, the flow of ions is a function about membrane potential, solvent concentration, radius of the electrode and electrode surface charge density on the human body skin. When the electro osmosis is the dominant factor, the flow of imported drugs can be described as follows ${ }^{[3]}$ :

$$
J_{i}=H^{\prime} D \frac{c z F}{R_{g a s} T} \frac{d \psi}{d x}
$$

The number of neutral molecules can be expressed as formula (3)

$$
t_{N}=\frac{W^{\prime} v C^{\prime} R_{g a s} T}{\frac{d \psi}{d x} F \sum_{i} c D Z_{i}^{2} H^{\prime}}
$$

Formula 4 show that $\mathrm{v}$ is a function of channel area and hole surface charge density. If the human body skin conductivity changes, the import drug flow will change too. Therefore, in order to make the drug flow constant, we have to stabilize skin conductivity. According to the experiment ${ }^{[4,5,6]}$ : human epidermal skin features (effective permeability ,effective hole area and hole charge density) can maintain a constant when conductivity constant.

\section{Ac pulse import system design}

According to the theory analysis, In the process of iontophoresis, we can obtain the voltage of the resistance in series with the skin of a human body by a single-chip microcomputer. According to the value of the sampling voltage, ac pulse voltage value imposed on the human body skin can be adjusted in real-time, so human epidermal skin conductivity can keep constant, so as to realize the constant of drug import flow.

To achieve the above purpose, ac pulse iontophoresis apparatus structure is as fig.1:

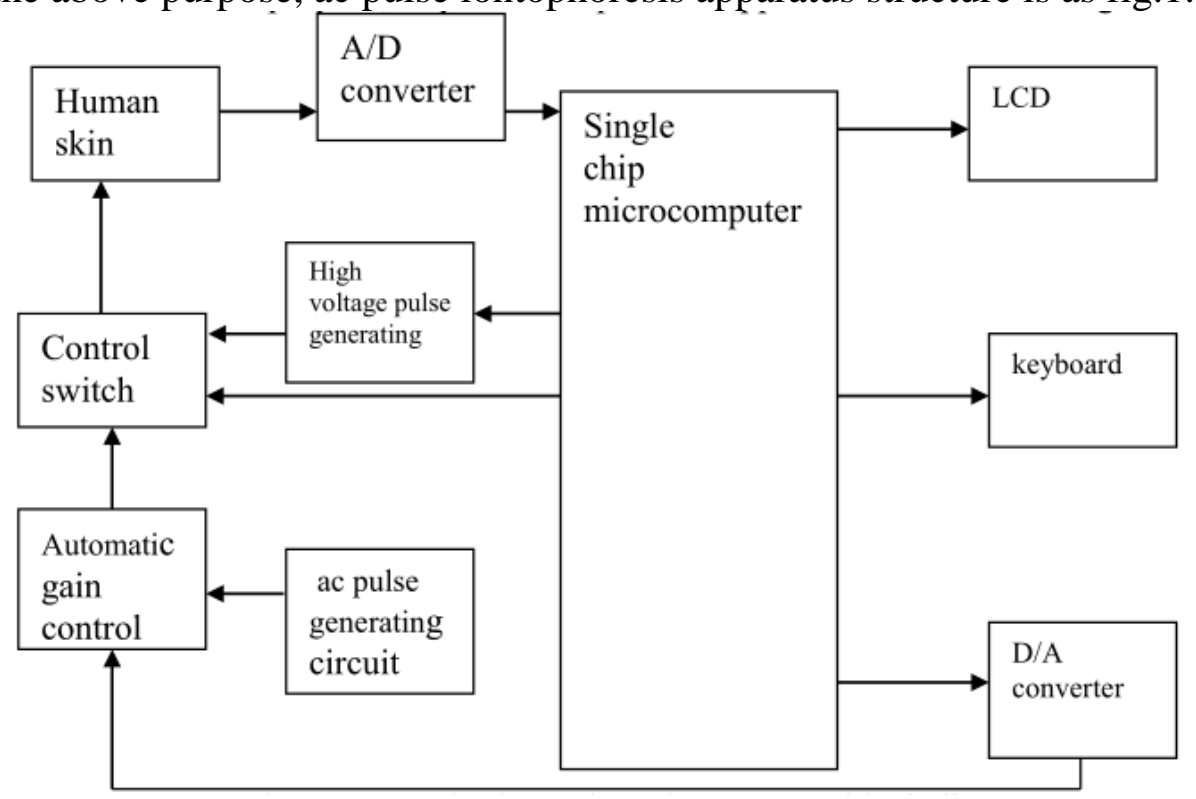

Figure 1 ac pulse iontophoresis apparatus block diagram

Ac pulse generating circuit. The circuit uses the wien bridge oscillator circuit, using the nonlinear of the diode to achieve automatic voltage stabilization. Regardless of the output signal in the positive half cycle or negative half cycle, there is always a diode forward conduction.

automatic gain control circuit Processing circuit will sample the output voltage of the automatic gain control circuit, and through the voltage value to control the control voltage of the automatic gain control circuit .Affected by the control voltage is the field effect transistor of the automatic gain control circuit, when the sample voltage value less than the reference value of processing circuit, the 
processing circuit output control voltage smaller, field-effect transistor resistance smaller, automatic gain control circuit of gain larger, so as to improve the output.

High pressure pulse and ac pulse switch control of import. In this project, the triode is adopted to control the effect time of electroporation, multi-channel analog switch is used to control the action time of ac pulse iontophoresis system.

Application software design

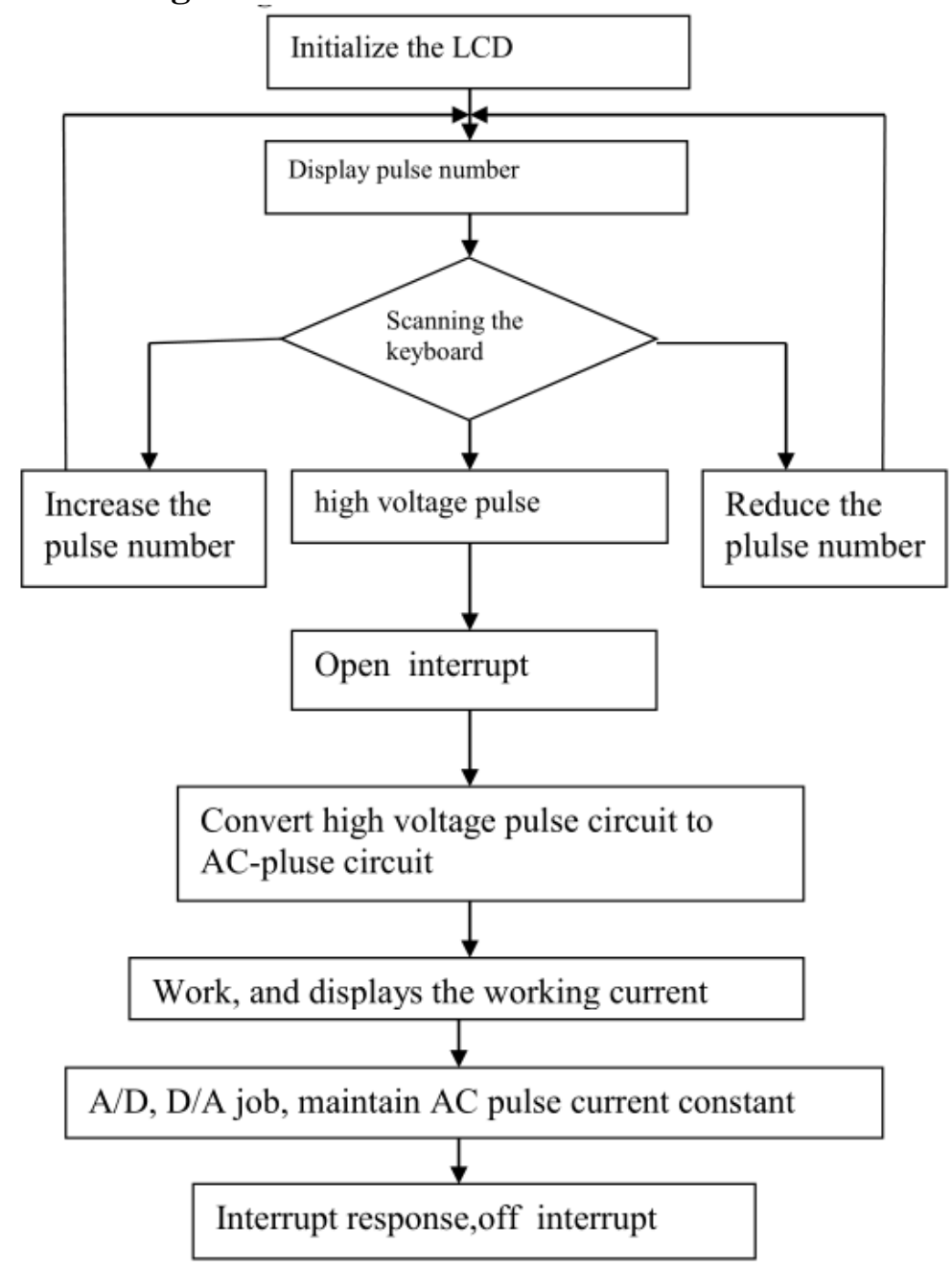

Figure 2 ac pulse iontophoresis instrument overall program flow chart

According to the hardware circuit, first of all, the single-chip microcomputer outputs high level to control the high voltage pulse generating circuit to work, at the same time control triode cut-off, and then the high voltage pulse effect the human body, after that the single-chip microcomputer outputs low level to make the triode conduction in order to forbid high voltage pulse generating circuit working.at the same time the analog switch switches on, then the ac pulse acts on the human body and $\mathrm{AD} / \mathrm{DA}$ converter set to sample the ac pulse amplitude in real-time. The single chip microcomputer will control the gain of the automatic gain control circuit through the sampling to ensure the ac pulse amplitude acting on human body skin basically stable, and then the skin membrane properties can keep stable and the import drug flow in human body basically constant. The total flow diagram as fig.2.

\section{Ac pulsed iontophoresis instrument experiment and data analysis}

The ac pulse iontophoresis system 'test. After applying high voltage pulse through the skin, the body's resistance to the skin is greatly reduced, high voltage pulse number is different, different human skin resistance loss. In this experiment, we can use the selection of human skin resistance for 
the $2 \mathrm{k}, 3 \mathrm{k}, 4 \mathrm{k}, 5 \mathrm{k}$ respectively to test the ac pulse output value's change along with the change of the sampling voltage. The results are as table 1.

Table 1 measurements when the human skin resistance value of $2 \mathrm{k}$

\begin{tabular}{|c|c|c|c|c|}
\hline $\begin{array}{l}\text { the original } \\
\text { resistance of } \\
\text { skin }(\mathrm{K} \Omega)\end{array}$ & $\begin{array}{l}\text { AD collection the } \\
\text { initial value( }(\mathrm{V})\end{array}$ & $\begin{array}{l}\text { Voltage output } \\
\text { values(V) }\end{array}$ & $\begin{array}{c}\text { Current } \\
\text { treatment(mA) }\end{array}$ & $\begin{array}{c}\text { Field effect } \\
\text { transistor } \\
\text { resistance }(\mathrm{K} \Omega)\end{array}$ \\
\hline 2 & 3.31 & 5 & 0.84 & 1.42 \\
\hline 2.3 & 3.10 & 5.2 & 0.83 & 1.12 \\
\hline 2.1 & 3.22 & 5.4 & 0.83 & 1.06 \\
\hline 1.9 & 3.35 & 5.1 & 0.85 & 1.41 \\
\hline 2 & 3.33 & 5.1 & 0.85 & 1.39 \\
\hline 1.9 & 3.33 & 4.9 & 0.84 & 1.45 \\
\hline 1.9 & 3.34 & 4.9 & 0.84 & 1.42 \\
\hline
\end{tabular}

Assume that every 10 minutes to measure a result, the measurement show that as the change of the human body resistance, voltage output value will automatically adjust, so as to ensure the treatment of relative constant current.

\section{Conclusions}

According to the measurement results, in the process of treatment, ac pulse voltage output will changes with sampling voltage, so as to ensure the constancy of the skin resistance, and then the import current constancy can achieve the goal of a stable drug import flow.

Through experimental analysis, ac pulse iontophoresis instrument can collect real-time import voltage, stable import drug flow, so as to provide a new method for treating process requires precise control of dosage, reduce the pain of patients.

\section{Acknowledgements}

This thesis is the project concluding paper .The project name: The Research of AC-Pulse Iontophoresis Apparatus, project department: The science and Technology Department of Hebei province, project number: 13272002.

\section{References}

[1] S.K. Li, K.D. Peck, A. -H. Ghanem and W.I. Higuchi: J. Pharm. Sci, Vol. 87 (1998) ,p40-48.

[2] W.M. Deen, ALChE. J.Vol.33(1987), p1409-1425.

[3] K.D. Peck, K. Hsu, S.K. Li , A.H.Ghanmen and W.I.Higuchi: J. Pharm. Sci, Vol. 87 (1998) , p1161-1169.

[4] S.K. Li, A.H.Ghanmen, W.I.Higuchi and K.D. Peck,: J. Pharm. Sci, Vol. 88 (1999) ,p419-427.

[5] H. Zhu, K.D. Peck, S.K. Li, A.H.Ghanmen and W.I.Higuchi: J. Pharm. Sci, Vol. 90 (2001) ,p932-942.

[6] H.Inada, A.H.Ghanmen and W.I.Higuchi: J. Pharm. Sci, Vol. 11 (1994) ,p687-697. 